\title{
De l'engagement au désenchantement (1945-1975) ? Jalons pour une histoire des intellectuelles catholiques
}

\section{Claire GUYOT}

\author{
(2) OpenEdition \\ Journals \\ Édition électronique \\ URL : https://journals.openedition.org/clio/140 \\ DOI : 10.4000/clio.140 \\ ISSN : 1777-5299 \\ Éditeur \\ Belin \\ Édition imprimée \\ Date de publication : 1 avril 2001 \\ Pagination : 155-166 \\ ISBN : 2-85816-577-7 \\ ISSN : 1252-7017
}

Référence électronique

Claire GUYOT, « De l'engagement au désenchantement (1945-1975) ? Jalons pour une histoire des intellectuelles catholiques », Clio. Histoire, femmes et sociétés [En ligne], 13 | 2001, mis en ligne le 19 juin 2006, consulté le 22 avril 2022. URL : http://journals.openedition.org/clio/140 ; DOI : https:// doi.org/10.4000/clio. 140

Ce document a été généré automatiquement le 22 avril 2022

Tous droits réservés 


\title{
De l'engagement au désenchantement (1945-1975)? Jalons pour une histoire des intellectuelles catholiques
}

\author{
Claire GUYOT
}

L'Église catholique a, tout particulièrement au XIX siècle, entretenu un rapport privilégié avec les femmes, magnifiant leur rôle de vierge, d'épouse ou de mère tout en limitant leur action à la sphère du privé ou du caritatif ${ }^{1}$. L'épanouissement de l'intelligentsia catholique dans la seconde moitié $\mathrm{du} \mathrm{xx}^{\mathrm{e}}$ siècle était susceptible de faire évoluer la situation puisque son principal projet consistait à redéfinir les questions nées de l'accélération de la sécularisation. Dans ce nouveau contexte, les femmes n'allaient-elles pas pouvoir prendre une nouvelle place? L'analyse d'itinéraires d'intellectuelles catholiques peut fournir des réponses. Elle permet de cerner l'importance de quelques figures féminines et de définir leur spécificité et leur originalité au sein du catholicisme et au sein de la société française. Malgré l'absence de travaux sur ces femmes, il est possible de répondre en partie à ces questions en examinant la place qu'a accordée le Centre catholique des intellectuels français (CCIF) à ces voix féminines. Créé en 1945 par des catholiques soucieux de confronter la foi à la modernité culturelle et convaincus de la nécessité d'établir un dialogue avec les différents courants de la pensée contemporaine, ce foyer de réflexion a trouvé très vite sa place au sein de l'intelligentsia parisienne. Carrefour de recherches, il joue collectivement un rôle de plate-forme de l'intelligence, analysant largement les grandes mutations de la société française. En trente ans, il réunit près de 2800 intervenants, venus d'horizons divers, pour des débats hebdomadaires, un rassemblement annuel et assure la publication d'une revue trimestrielle ${ }^{2}$. Il constitue donc un baromètre de la vie intellectuelle française et un instrument utile pour étudier la place de ces voix féminines. 
2 Si les intellectuels catholiques s'affirment comme groupe constitué cinquante ans après l'affaire Dreyfus ${ }^{3}$, c'est en grande partie à une initiative féminine qu'ils le doivent. Le Centre catholique des intellectuels français, qui a légitimé l'expression «intellectuel catholique », a en effet été fondé par Madeleine Leroy, une sévrienne qui appartenait à la Congrégation des Filles du Cœur de $\mathrm{Marie}^{4}$. Figure originale que celle de cette "religieuse cachée ${ }^{5}$ qui, après avoir créé un foyer de réflexion et lui avoir donné une structure administrative, prit une place importante dans son organisation matérielle et intellectuelle tout en refusant de jouer un rôle public. Entre-t-elle pour autant dans la catégorie des intellectuelles? La réponse est négative si l'on retient les critères traditionnellement admis pour définir l'intellectuel (l'engagement politique ou l'expertise ${ }^{6}$ ) et ce, bien qu'elle accepte d'être nommée vice-présidente du Centre catholique des intellectuels français ${ }^{7}$ et de rédiger un manifeste dénonçant l'arrestation abusive d'un journaliste catholique ${ }^{8}$. Madeleine Leroy semble même le contraire d'une intellectuelle : malgré ses compétences (elle est cacique de l'École normale supérieure de Sèvres et membre du jury d'agrégation de mathématiques), elle délaisse la carrière universitaire pour l'enseignement secondaire ${ }^{9}$; au sein $\mathrm{du}$ CCIF, elle ne prend part à aucun débat public et ne rédige aucun article pour la revue qu'elle a contribué à développer et à soutenir financièrement. La réponse est positive si l'on prend en compte un autre critère, celui d'un engagement public dont les conséquences sont durables ${ }^{10}$. Le souci de faire dialoguer christianisme et modernité conduit en effet Madeleine Leroy à constituer un organisme qui revendique publiquement le concept d'intellectuel catholique. Si ce choix peut être perçu comme un prolongement du travail de catéchisme qui fut confié traditionnellement par l'Église aux femmes, il s'en éloigne profondément par la visibilité que souhaita la fondatrice.

3 Madeleine Leroy n'est pas la seule femme à s'investir dans l'organisation des activités intellectuelles du nouveau foyer. Une jeune lyonnaise, Odette Laffoucrière ${ }^{11}$, participe de ce même type d'engagement. Invitée en 1947 par l'assistant ecclésiastique, l'abbé Émile Berrar, cette ancienne responsable nationale de la Jeunesse agricole catholique pour la culture populaire s'investit considérablement et contribue fortement à écarter la tendance institutionnelle et représentative du foyer au bénéfice de la seule dimension réflexive ${ }^{12}$. Célibataire, elle voue sa vie à la recherche philosophique et l'envisage comme une vocation à part entière. À la différence de Madeleine Leroy, sa modeste participation publique n'est due qu'à sa carence de diplômes universitaires. Souffrant de cette situation, elle part au début des années 1950 en Allemagne pour préparer une thèse sur Martin Heidegger, sous la direction de Paul Ricœur.

4 L'une et l'autre incarnent donc un premier profil d'intellectuelle catholique : elles participent du modèle religieux de l'engagement mais s'en détachent par l'originalité de leur démarche et leur indépendance d'esprit. De fait, leurs amitiés théologiques (le père de Grandmaison et le père de Montcheuil pour Madeleine Leroy, l'abbé Daniel Pézeril pour Odette Laffoucrière ${ }^{13}$ ) les conduisent à se dégager d'un enseignement doctrinal étroit et surtout à chercher de nouveaux espaces d'expression où l'accueil de la pensée agnostique est fondamental.

5 En créant le Centre catholique des intellectuels français, Madeleine Leroy rend en outre possible l'émergence d'un deuxième profil d'intellectuelle catholique, celui d'experte. Des femmes participent en effet aux activités du Centre en collaborant à la revue, aux colloques et aux débats. Elles ont suivi un itinéraire universitaire classique, ont souvent intégré une École normale supérieure, la plupart ont passé l'agrégation, et certaines, 
plus rarement, ont soutenu une thèse ${ }^{14}$. Comme leurs pairs masculins, elles manifestent le souci de travailler à la modernisation du dépôt de la foi et à la respectabilité d'une pensée catholique pluraliste, ouvertement en phase avec les connaissances du moment. Elles participent ainsi de cet esprit d'ouverture qui traverse une bonne partie du catholicisme français et qui conçoit une présence au monde dans le respect de l'altérité. Certaines d'entre elles répondent ainsi à l'invitation de leurs anciens aumôniers catholiques (tout particulièrement le père Yves de Montcheuil et le père Jean Daniélou) qui leur rappelaient le devoir de présence et de réflexion dans la société ${ }^{15}$. Leur position ne se différencie aucunement de celle des intellectuels catholiques et leur statut de femmes n'induit aucune réflexion sur la vocation féminine. Cette modeste communauté de travail trouve en partie sa source dans la mixité des groupes de réflexion qui se sont organisés pendant la guerre: scientifiques qui se rassemblent pour témoigner de la compatibilité entre la foi chrétienne et la recherche au-delà d'un fidéisme qui sépare radicalement convictions religieuses et activités professionnelles ${ }^{16}$; philosophes qui tentent de se dégager d'un thomisme étroit dominé encore par la perspective antimoderniste ${ }^{17}$. Elle naît également du vivier inestimable que constitue la Paroisse universitaire qui rassemble depuis l'entre-deux-guerres les enseignants catholiques de l'enseignement public ${ }^{18}$.

6 La visibilité de l'intellectuelle catholique s'accomplit donc au milieu des années 1940, au sein du Centre catholique des intellectuels français et accompagne celle de son confrère masculin ${ }^{19}$. Si leur nombre reste modeste ${ }^{20}$, il l'est davantage en raison de la condition générale des femmes dans l'intelligentsia française ${ }^{21}$ qu'à une réticence de la hiérarchie catholique. L'expression publique des femmes n'est pas contestée par le Magistère romain, mais leurs réflexions - comme celle des hommes - sont sous surveillance, les laïcs catholiques étant avant tout considérés comme des auxiliaires de la théologie. Au début de la décennie 1960, l'accélération de la sécularisation tout particulièrement marquée par le renouvellement des questions féminines ${ }^{22}$ était susceptible de contribuer à l'émergence d'un troisième profil d'intellectuelle catholique.

7 En ces années, le CCIF connaît une féminisation de son équipe de direction. Prolongeant l'action de Madeleine Leroy, des femmes participent en plus grand nombre à l'orientation intellectuelle du foyer de réflexion. C'est le cas tout particulièrement de Renée Bédarida, épouse du nouveau secrétaire, François Bédarida ${ }^{23}$. Le couple s'entoure d'une petite équipe de travail composée presque uniquement d'hommes mais où prennent place Odette Laffoucrière - revenue d'Allemagne ${ }^{24}$ - et Suzanne Villeneuve. Cette dernière est présente depuis le début des années 1950 comme trésorière. Amie de Madeleine Leroy, agrégée de philosophie, elle enseigne en lycée, puis devient assistante à l'université de Nanterre. Claude Imbert, ancienne élève de l'École normale supérieure de la rue d'Ulm et « Princesse tala » est invitée, quant à elle, au comité de rédaction. À la fin des années 1960, Aline Coutrot, une agrégée d'histoire rejoint l'équipe sur la demande de son ancien directeur de thèse, René Rémond, le nouveau président du CCIF ; puis c'est au tour d'une génération plus jeune de se joindre au noyau dirigeant : anciens du Centre Richelieu (aumônerie de la Sorbonne) comme Claudine Vey (qui vient accompagnée de son mari) et membres de la Paroisse universitaire comme Nicole Desmerger. En 1968, Suzanne Villeneuve devient rédactrice en chef de la revue du Centre $^{25}$. 
8 Cette féminisation de l'équipe de direction s'accompagne surtout d'une plus grande présence d'oratrices ${ }^{26}$ et d'un intérêt grandissant pour les questions féminines et la morale conjugale. Entre 1960 et 1966, deux numéros de la revue, une séance de la Semaine des intellectuels catholiques et plusieurs débats y sont consacrés ${ }^{27}$. Le premier numéro sur la sexualité et la limitation des naissances est publié en $1963^{28}$, le deuxième sur «Femme : nature et vocation", quelques mois plus tard ${ }^{29}$. Le CCIF suit donc le parcours de cette génération de femmes qui croit l'égalité entre les sexes acquise et qui analyse la condition des femmes à travers leurs vécus privé et professionnel ${ }^{30}$. Si Renée Bédarida ne prend pas position publiquement (elle rédige très peu d'articles et ne signe aucun manifeste ${ }^{31}$ ), elle est un vecteur essentiel de cette mise en débat et constitue avec son mari une sorte de secrétariat à deux têtes. Comme pour Madeleine Leroy, la clef de voûte de son engagement trouve son origine dans la volonté de faire dialoguer christianisme et connaissances du moment. Mais désormais ce sont les enjeux des sciences humaines et les nouvelles expressions de la modernité qui sont au cœur de la réflexion.

10 La présence grandissante des intellectuelles catholiques au sein du CCIF ne s'accompagne pas paradoxalement de prises de position publiques sur les questions féminines. Si des catholiques viennent s'exprimer sur ces questions, la majeure partie d'entre elles ne sont pas des intellectuelles mais des médecins comme la doctoresse Odile Cordier qui participe aux activités du CLER (Centre de liaison des équipes de recherche) ou des militantes comme Marguerite Lambert très active dans le CMR (Chrétiens en monde rural). Quant aux intellectuelles, elles sont ... protestantes: Évelyne Sullerot, une des fondatrices de la Maternité heureuse ou encore Francine Dumas, psychologue très engagée dans le mouvement féministe. Cette absence (alors que les clercs sont nombreux à s'exprimer sur ces sujets ${ }^{32}$ ) est caractéristique de la modestie des intellectuelles catholiques sur ce terrain moral. En ces années, le catholicisme romain reste largement imprégné d'une vision intransigeante avec un système de pensée fondé sur une vision pyramidale et hiérarchique. Il envisage d'assez mauvaise grâce le rôle que cherchent à prendre les laïcs dans la formulation dogmatique et s'il se réjouit de l'entrée de la femme dans la vie publique et dans le monde de la pensée, il les encourage parallèlement à poursuivre leur vocation de mère et d'épouse. L'encyclique Humanae vitae publiée durant l'été 1968, sous le pontificat de Paul VI, qui condamne toute forme contraceptive ${ }^{33}$ provoque d'ailleurs une accélération de la prise de distance des femmes à l'égard de l'institution ecclésiale.

11 L'Église catholique en voulant «contrôler le ventre des femmes » et en les excluant de l'élaboration du discours théologique et des fonctions sacerdotales conduit de nombreuses femmes à s'éloigner ${ }^{34}$. En 1996, le journaliste Henri Tincq s'interrogeait dans Le Monde sur "Le silence des intellectuels catholiques " ${ }^{35}$. Quelques jours plus tard Françoise Praderie, astronome à l'observatoire de Paris et ancienne de l'Union catholique des scientifiques français précisait :

(...) l'année 1968 est aussi celle de la publication de l'encyclique Humanae vitae. Avec ce texte se sont écroulées bien des solidarités, en particulier celles qui s'élaboraient au sein du Centre catholique des intellectuels français, avec les scientifiques - j'en suis - réfléchissant aux apports de la recherche scientifique (...). Nombre de laïcs exerçant des professions intellectuelles ou scientifiques ou politiques, et qui ont suivi des itinéraires semblables au mien, n'ont plus envie de se battre pour faire que l'Église cesse de tenir des discours d'autorité et commence à 
regarder autour d'elle, avec les meilleurs outils de la réflexion, avant qu'il soit trop $\operatorname{tard} .^{36}$

12 Si un lien privilégié s'est noué entre féminisme et religion réformée ${ }^{37}$, il n'en a donc pas été de même pour le catholicisme. Au contraire, l'émergence des questions sur les droits des femmes a accentué le désenchantement face à une Église jugée autoritaire, masculine et trop attachée à son inculturation gréco-latine ${ }^{38}$. Au sein du Centre catholique des intellectuels français, des femmes continueront leur travail d'experte tout en se refusant à prendre position sur les problèmes de la société ${ }^{39}$. D'autres, mais en dehors du CCIF élaboreront les prémices d'une théologie féministe. Tel est le cas de Françoise Vandermeersch qui ouvre sa revue Échanges à des intellectuels et des militants et consacre de nombreux numéros aux questions féminines ${ }^{40}$.

Si en cette seconde moitié $d u x^{e}$ siècle les intellectuels catholiques se déclinent encore majoritairement au masculin, des femmes n'en sont pas moins présentes comme le montre l'histoire $\mathrm{du}$ Centre catholique des intellectuels français. Parmi ces intellectuelles très rares sont celles qui ont mis leurs compétences et leur notoriété au service d'une cause politique ou sociale. La plupart préfèrent le terrain de l'expertise et participent ainsi à la réintégration du catholicisme dans le monde de la pensée. Lorsque la question des femmes s'affirme vivement dans la société française, les intellectuelles catholiques ne s'en font pas les porte-voix. Au contraire, désabusées par l'intransigeance et l'autoritarisme de l'Église catholique sur les questions morales, elles préfèrent se désengager. De fait, nées d'un double déblocage franco-romain, les intellectuelles catholiques s'effacent avec le retour d'un blocage romain ${ }^{41}$. Se trouvant à l'interface du culturel et du religieux, ces intellectuelles subissent les contraintes substantielles à leur spécificité de croyantes: demeurer fidèles à l'Église tout en conservant autonomie et droit d'expression.

\section{BIBLIOGRAPHIE}

Aubert Jean-Marie, 1975, Antiféminisme et christianisme, Cerf-Desclée.

CHAPERON Sylvie, 1998, « 1945-1970, reprendre l'histoire du féminisme », in L'histoire sans les femmes est-elle possible?, Perrin, pp. 205-215.

--, janvier-mars 2000a, « Le Mouvement Jeunes Femmes 1946-1970 : de l'Évangile au féminisme », Bulletin de la Société d'histoire du protestantisme, tome 146, pp. 153-183.

--, 2000b, Les années Beauvoir, 1945-1970, Fayard.

Charle Christophe, 1999, «Les femmes dans l'enseignement supérieur. Dynamiques et freins d'une présence 1946-1992 ", in Vincent Duclert, Rémi Fabre, Patrick Fridenson (dir.), Avenirs et avant-gardes en France, $\mathrm{XIX}^{e}$-XX ${ }^{e}$ siècles. Hommage à Madeleine Rebérioux, pp. 83-105.

Colin Pierre, 1997, L'audace et le soupçon. La crise du modernisme dans le catholicisme français (1893-1914), DDB. 
Dumoulin Olivier, 1998, « Archives au féminin, histoire au masculin, les historiennes professionnelles en France, 1920-1965 », in Anne-Marie Sohn et Françoise Thélamon (dir.), L'histoire sans les femmes est-elle possible?, pp. 343-356.

Duquoc Christian, « Théologie », Catholicisme, hier, aujourd'hui, demain, dir. Jacquemet.

Foucault Michel, 1994, Dits et écrits, tome 3, 1976-1979, Paris, Gallimard.

Fouilloux Étienne, 1995, Yves de Montcheuil, philosophe et théologien jésuite (1900-1944), Média Sèvres. --, janvier-mars 1997, « "Intellectuels catholiques” ? Réflexions sur une naissance différée », Vingtième siècle. Revue d'histoire, 53, pp. 13-24.

--, 1998, Une Église en quête de liberté. La pensée catholique entre modernisme et Vatican II, Paris, DDB. Guyot Claire, juillet-décembre 1998, « La naissance du Centre catholique des intellectuels français, 1941-1951 », Revue d'histoire de l'Église de France, pp. 323-336.

--, juillet-septembre 1999, « Entre morale et politique : le CCIF et la décolonisation », Vingtième siècle. Revue d'histoire, pp. 75-86.

--, 2000, « Modernité et christianisme. Le Centre catholique des intellectuels français (1941-1976). Itinéraire collectif d'un engagement », Université Lumière Lyon II, thèse inédite. Jacquin Françoise, 1987, L'histoire du Cercle Saint-Jean-Baptiste : l'enseignement du père Daniélou, Beauchesne.

Langlois Claude, 1995, « Toujours plus pratiquantes. La permanence du dimorphisme sexuel dans le catholicisme français contemporain », Clio Histoire Femmes et Sociétés, 2, pp. 229-260.

Ory Pascal et Sirinelli Jean-François, 1986, Les intellectuels de l'affaire Dreyfus à nos jours, Paris, A. Colin.

Prost Antoine, septembre 1998, «Changer le siècle », Vingtième siècle. Revue d'histoire, pp. 14-26.

Rochefort Florence, janvier-mars 2000, « Féminisme et protestantisme au xixe siècle, premières rencontres 1830-1900 », Bulletin de la Société d'histoire du protestantisme, tome 146, pp. 69-89.

Quere France, 1976, La femme avenir, Le Seuil.

Schlegel Jean-Louis, mai 2000, « Intellectuels catholiques : silences contraints, silences voulus », Esprit, pp. 86-102.

Sevegrand Martine, 1995, Les enfants du bon Dieu, les catholiques français et la procréation au $\mathrm{Xx}^{e}$ siècle, Albin Michel.

Dans la tourmente révolutionnaire, les Filles du Cœur de Marie, 1989, éditions Lambert-Laurent S.A.

\section{NOTES}

1.Langlois 1995 .

2.Guyot 2000.

3.Fouilloux 1997.

4.Madeleine Leroy est née dans un milieu aisé, elle suit les traces de ses deux aînés : un frère qui fut proviseur du lycée Condorcet et une sœur sévrienne agrégée de philosophie. Elle choisit les mathématiques et passe l'agrégation en 1925. Sur Madeleine Leroy et son rôle dans la création du CCIF, voir Guyot 1998 et 2000. 
5.La vocation de cette congrégation née sous la Révolution française est de vivre incognito dans le monde, sans signe distinctif et sans résidence commune obligatoire. Dans la tourmente révolutionnaire, les Filles du Coeur de Marie, 1989.

6.Pascal Ory et Jean-François Sirinelli pour le premier type (Pascal Ory et Jean-François Sirinelli 1986) et Michel Foucault pour le second (Michel Foucault 1994 : 112-113). 7.Elle le gardera jusqu'à son départ en 1959 pour Tokyo lorsqu'elle se lance dans la création d'un foyer pour jeunes filles. Elle y décédera d'un cancer dès 1963.

8.Fortement investi dans le mouvement de libération de l'Algérie, Robert Barrat était l'ancien secrétaire général du CCIF. Il avait été arrêté par les autorités françaises le 26 septembre 1955 pour avoir fait paraître un article sur un chef algérien rebelle. Lettre de protestation écrite par Étienne Borne, Lionel Assouad et Madeleine Leroy 26 septembre 1955, p. 1. Archive du Centre catholique des intellectuels français.

9.Un comportement typique de la génération des diplômées du milieu des années 1930. 10.Sur l'engagement et ses différentes expressions, voir Prost 1998.

11.Ancienne élève d'Henri-Irénée Marrou, elle quitte Lyon après la guerre pour passer le concours de bibliothécaire et travaille pour Alfred Sauvy. Témoignage à l'auteur, 2 février 1994.

12.Le Centre catholique des intellectuels français rassemblait tous les diplômés catholiques de l'enseignement supérieur, certains de ses membres souhaitaient donc voir développer cette dimension de représentativité et d'apostolat auprès des groupements de diplômés. Voir sur cette question Guyot $2000: 130-146$.

13.Le père de Grandmaison était le directeur de la revue Études, le père de Montcheuil était professeur de théologie à l'Institut catholique de Paris ; l'abbé Daniel Pézeril était professeur au Séminaire des Carmes, puis fut chargé d'une paroisse parisienne. Tous les trois voulaient rendre pensable le christianisme à leurs contemporains et appelaient l'Église à prendre en compte les nouveaux champs de recherche scientifiques.

14.Citons en quelques-unes : Jeanne Delhomme, qui enseigne à l'université de Poitiers, défend une ligne existentialiste entre Gabriel Marcel et Jean-Paul Sartre ; Geneviève Lewis-Rodis propose une réflexion d'historienne de la philosophie ; la biologiste Henriette Alimen défend la théorie évolutionniste encore mal acceptée par la hiérarchie catholique.

15.Yves de Montcheuil était aumônier de la Jeunesse étudiante chrétienne féminine, Jean Daniélou, celui des sévriennes. Voir pour le premier Fouilloux 1995 et pour le second, Jacquin 1987.

16.Sous l'Occupation, de jeunes normaliens avaient souhaité créer des équipes de travail. Ils demandèrent à Madeleine Leroy de les aider à faire aboutir leur projet. Après la guerre, ils se réunirent au sein de l'Union catholique des scientifiques français (UCSF), union associée au CCIF.

17.Colin 1997.

18.Madeleine Leroy était d'ailleurs secrétaire générale de la Paroisse universitaire lorsqu'elle créa le CCIF.

19.Les intellectuels catholiques présentent de ce point de vue une originalité certaine par rapport aux autres intellectuels en dédaignant massivement l'engagement politique tel que Jean-Paul Sartre l'incarne.

20.En trente ans, sur les 2800 personnes sollicitées, seulement deux cent quarante-cinq femmes se sont exprimées publiquement, soit un peu moins de $9 \%$.

21.Charle 1999 : 83-105 et Dumoulin $1998:$ 343-356.

22. Chaperon 2000. 
23.Normalien, agrégé d'histoire, François Bédarida est choisi comme secrétaire général du CCIF en septembre 1960, il remplace alors Étienne Borne. Il reste à ce poste jusqu'en 1966 date de son départ pour la Grande-Bretagne. Renée Bédarida a participé aux mouvements de résistance lyonnaise en diffusant les premiers cahiers de Témoignage chrétien. Sans choisir une carrière universitaire, elle rédige plusieurs ouvrages historiques. Entretiens avec l'auteur, 9 mars 1994 et 15 mai 1997.

24.Elle quitte peu de temps après le Centre jugeant les activités intellectuelles insuffisamment orientées sur les questions philosophiques. Elle fonde avec l'abbé Daniel Pézeril les conversations de Saint-Jacques du Haut-Pas.

25.Étienne Fouilloux lui succède en 1971, puis après le départ de ce dernier en 1973, elle reprend le poste jusqu'à la disparition du CCIF en 1976.

26.44 femmes venues entre 1951 et 1957, 97 entre 1958 et 1965 et 103 entre 1965 et 1975. Philosophes comme Georgette Vignaux, femme du médiéviste et syndicaliste Paul Vignaux ou encore Clémence Ramnoux, spécialiste des pré-socratiques.

27.L'équipe suivante (1966-1976) s'intéresse surtout à la morale conjugale et délaisse les autres questions, tout particulièrement le travail des femmes et ses enjeux.

28.Le cahier regroupe les signatures de Marguerite Lambert, Madame Lieury, des docteurs Abiven, Debray, Le Moal, Sutter et des pères Beirnaert, Bouchaud, de Contenson, Dubarle et de Lestapis. Les orateurs invités sont donc équitablement partagés entre ceux qui condamnent toute contraception artificielle et ceux qui y sont plutôt favorables. Recherches et Débats 43, Fayard, juin 1963. Sur ces questions de morale conjugale, voir Sèvegrand, 1995 et Guyot 2000 : 421-426.

29.Recherches et Débats 45, Fayard, décembre 1963. Ce numéro rassemble les contributions de M.-J. Chombart de Lauwe, A.-M. Rocheblave-Spenlé, le docteur Denard-Toulet, S. Villeneuve, J.-L. Gardies, les pères Tilliette et Orrieux. Le cahier fait le point sur les conflits et les malaises pour dégager des profils féminins différents, il souligne la vocation particulière de la femme tout en s'interrogeant sur l'existence d'une spécificité chrétienne.

30.Chaperon 1998.

31.Un article sur la condition des Nord-Africains (publié dans Recherches et Débats 47, juin 1964) et surtout elle prépare le numéro spécial consacré à l'histoire du CCIF depuis 1945 (Recherches et Débats 54, avril 1966).

32.Le jésuite Louis Beirnaert, l'abbé Marc Oraison et le chanoine Pierre de Locht. 33. Sur cette question voir Sèvegrand $1995:$ 291-364.

34.En 1975, le père Jean-Marie Aubert fait paraître Antiféminisme et christianisme, ouvrage qui souligne non seulement les raisons pour lesquelles l'Église catholique est restée un bastion de la masculinité, mais aussi les responsabilités chrétiennes dans la formation et l'évolution de l'antiféminisme occidental.

35.Le Monde 15 mars 1996.

36.Le Monde 9 avril 1996.

37.Chaperon janvier-mars 2000 et Rochefort janvier-mars 2000.

38.Voir la mise au point de Jean-Marie Aubert et l'article de Christian Duquoc "Théologie ».

39.Annie Jaubert, une spécialiste des origines chrétiennes, vient ainsi plusieurs fois.

40.Françoise Vandermeersch appartenait à la congrégation des auxiliatrices du Purgatoire. Fortement marquée par le mouvement de Mai 1968, elle s'engage dans un catholicisme contestataire. Tout en dirigeant une revue, elle refuse le titre d'intellectuelle catholique. À la fin des années 1960, elle consacre plus de vingt 
numéros d'Échanges à la place de la femme dans l'Église. Je remercie Sabine Rousseau pour ces informations.

41.Sur la crise de l'intelligentsia catholique, voir Schlegel mai $2000: 86-102$ et Guyot

2000 : 467-513.

\section{RÉSUMÉS}

L'analyse d'itinéraires féminins à travers l'histoire du Comité Catholique des Intellectuels Français (CCIF) met en valeur trois profils d'intellectuelles catholiques qui cherchent à résoudre les interrogations posées à l'intelligence croyante par les derniers développements de la connaissance. La majeure partie de ces femmes forment ainsi un groupe d'expertes qui refusent de s'engager au service d'une cause politique ou sociale. Elles sont donc bien éloignées du modèle intellectuel qui prévaut alors et qu'incarne Jean-Paul Sartre.

Thise analysis of women's involvement in the Catholic Committee of French Intellectuals (CCIF) highlights the profiles of three Catholic intellectual women who sought to resolve the questions posed to the believer's intelligence by the latest developments of knowledge. Most of these women thus constitute a group of experts who refused to commit themselves to the service of a political or social cause. They are therefore far removed from the then-dominant intellectual model incarnated by Jean-Paul Sartre.

\section{AUTEUR}

\section{CLAIRE GUYOT}

Claire GUYOT est agrégée d'histoire. Elle a soutenu en 2000 une thèse consacrée aux intellectuels catholiques dans la seconde moitié du XX siècle : « Modernité et christianisme. Le Centre catholique des intellectuels français (1941-1976). Itinéraire collectif d'un engagement », Université Lumière Lyon II, sous la direction d'Étienne Fouilloux. Elle a publié : « La naissance du Centre catholique des intellectuels français, 1941-1951 ", Revue d'histoire de l'Église de France, juillet-décembre 1998, pp. 323-336 et « Entre morale et politique: le CCIF et la décolonisation », Vingtième siècle. Revue d'histoire, juillet-septembre 1999, pp. 75-86. 